\title{
Funções Executivas: Correlação entre Dois Inventários e Desempenho Acadêmico em Matemática
}

\author{
Cristiane Marx Flor ${ }^{1}$ (D), Ana Paula Soares de Campos (D), Suzete Araujo Aguas Maia (D), Alessandra Gotuzo Seabra (D) \\ Universidade Presbiteriana Mackenzie, São Paulo-SP, Brasil
}

\section{RESUMO}

Diante da importância das funções executivas para o desempenho escolar, investigou-se a associação entre notas na disciplina de matemática e desempenho em funções executivas, conforme relato de pais e professores de alunos do $5^{\circ}$ ano, nos instrumentos BRIEF e IFERA-I. Participaram do estudo pais/responsáveis e professores de 47 crianças, com idade entre 10 e 11 anos, de uma escola particular do estado de São Paulo. Houve correlação significativa entre notas na disciplina de matemática e avaliações de pais e professores no BRIEF e IFERA-I, sendo que o BRIEF apresentou maiores correlações que o IFERA-I. Respostas dos professores apresentaram correlações mais altas com desempenho matemático do que as dos pais em todas as escalas, exceto na organização de materiais do BRIEF, e os pais relataram mais dificuldades do que os professores. Os resultados corroboram a associação entre funções executivas e matemática, bem como a importância de múltiplos informantes na avaliação infantil.

Palavras-chave: funções executivas; BRIEF; IFERA-I; matemática.

ABSTRACT - Executive Functions: Correlation Between Two Inventories and Academic Performance in Mathematics Considering the importance of executive functions for school performance, the present study investigated the association between grades in Mathematics and performance in executive functions, as reported by parents and teachers of 5th grade students, using the BRIEF and IFERA-I instruments. Participants were parents/guardians and teachers of 47 children, aged between 10 and 11 years, from a private school in the state of São Paulo. There was significant correlation between grades in Mathematics and the parents' and teachers' assessments in the BRIEF and IFERA-I, with the BRIEF presenting higher correlations than the IFERA-I. The teachers' responses showed higher negative correlations with mathematical achievement than those of the parents in all subscales, except in the organization of materials (BRIEF), and the parents reported more difficulties than the teachers. The results corroborate the association between executive functions and Mathematics, as well as the importance of multiple informants in child assessments.

Keywords: executive-functions; BRIEF; IFERA-I; Mathematics.

RESUMEN - Funciones Ejecutivas: Correlación entre dos Inventarios y Rendimiento Académico en Matemáticas Ante la importancia de las funciones ejecutivas para el rendimiento escolar, el presente estudio investigó la asociación entre las notas en matemáticas y el rendimiento en funciones ejecutivas a través de los instrumentos BRIEF e IFERA-I, tomando como indicativo los informes de padres y profesores de alumnos del $5^{\circ}$ año. Participaron del estudio padres/tutores y profesores de 47 niños, con edades comprendidas entre 10 y 11 años, de una escuela privada de la provincia de São Paulo. Los resultados mostraron que existe correlación negativa significativa entre las notas en la asignatura y las evaluaciones de padres y profesores en el BRIEF e IFERA-I, siendo que el BRIEF presentó mayores correlaciones que el IFERA-I. Las respuestas de los profesores obtuvieron correlaciones más altas con el rendimiento matemático que las de los padres en todas las escalas, excepto en la organización de materiales (BRIEF), o sea, los padres parecen haber sido más rigurosos en la evaluación del comportamiento de sus hijos.

Palabras clave: funciones ejecutivas; BRIEF; IFERA I; matemáticas.

Para aprender, raciocinar e solucionar problemas, o ser humano faz uso de uma gama de diferentes habilidades cognitivas. O conjunto das habilidades que permite $o$ controle, supervisão e regulação do próprio pensamento e comportamento é conhecido como funções executivas (FE) (Baggetta \& Alexander, 2016; Clements, Sarama, \& Germeroth, 2016). Por meio das FE, é possível responder de forma flexível ao ambiente e engajar-se em pensamento e ações intencionais, direcionadas a um objetivo (Cragg \& Gilmore, 2014). Embora tenha havido divergências sobre a uni ou multidimensionalidade do construto FE (Miyake et al., 2000), atualmente há consenso de que o termo se refere a um conjunto de funções inter-relacionadas com capacidade de organizar, planejar, automonitorar, inibir respostas, flexibilizar pensamentos e manipular informações de forma temporária na 
memória (Carim, Miranda, \& Bueno, 2012). Outro consenso entre autores é que FE não são exclusivas do domínio cognitivo, mas também pertencem aos domínios socioemocionais e comportamentais da performance humana (Baggetta \& Alexander, 2016). Há algumas divergências acerca de quais componentes estão envolvidos nas $\mathrm{FE}$, sendo que um dos modelos mais robustos considera a inclusão de três componentes básicos centrais, distintos, mas relacionados, quais sejam, o componente de atualização da memória de trabalho, a inibição de respostas e a alternância (Miyake \& Friedman, 2012) ou, no modelo de Diamond (2013), a memória de trabalho, a inibição e a flexibilidade cognitiva.

O período pré-escolar, entre 2 e 5 anos de idade, é considerado um período sensível de desenvolvimento das FE, por conta de importantes mudanças no córtex pré-frontal, embora o processo como um todo prossiga com crescente complexidade ao longo da adolescência e início da idade adulta (Zelazo \& Muller, 2011). De acordo com Zelazo e Muller (2002), a organização das FE sofre mudanças no decorrer do processo de desenvolvimento, de modo que as FE são um construto relativamente indiferenciado em crianças pequenas e torna-se mais modular com o aumento da idade.

FE correlacionam-se com diversos desfechos ao longo da vida, incluindo o desempenho acadêmico de alunos (Zimmerman, 2002). Segundo Clements et al. (2016), as FE afetam o desempenho da criança na escola de duas formas: possibilitando o desenvolvimento e o uso dos processos cognitivos envolvidos na aprendizagem escolar e possibilitando às crianças atentar às regras escolares e aprender em contextos sociais variados. De forma mais específica, mensurações em FE têm sido associadas ao desempenho em matemática e leitura (Blair, Protzko, \& Ursache, 2011; Van Der Ven, 2011), embora um número maior de estudos tenha mostrado FE mais fortemente associadas à matemática do que à leitura ou linguagem (Blair et al., 2011; Monnete, Bigras, \& Guay, 2011). Estudos longitudinais também sugerem que as FE favorecem a aquisição de novas habilidades matemáticas (Blair \& Razza, 2007; Passolunghi \& Lanfranchi, 2012). Segundo Vitterbori et al. (2015), entre uma variedade de processos cognitivos que têm sido associados como preditores de habilidades matemáticas, as FE mostram especial importância, particularmente, a memória de trabalho. Além dela, a função de controle inibitório parece possuir um papel importante no desempenho inicial em matemática (Blair \& Razza, 2007). Pesquisando crianças com desempenho inferior em matemática, Lan et al. (2011) também identificaram déficits específicos nos processos de inibição e memória de trabalho.

Embora as correlações significativas encontradas possam sugerir uma relação causal entre FE e desempenho escolar na matemática, é importante destacar que os estudos até a presente data não mostram relações de causalidade (Clements et al., 2016). Na verdade, é possível que haja uma relação bidirecional entre FE e desempenho em matemática, uma predizendo parcialmente a outra (Van Der Ven, 2011), ou seja, a proficiência no desempenho matemático inicial também poderia predizer o desenvolvimento de futuros processos de FE. Ainda segundo Clements et al. (2016), uma relação causal entre FE e desempenho acadêmico só poderia ser testada caso fossem utilizados outros métodos de delineamento experimental, com grupos de controle, distribuição aleatória ou análises de regressão.

Uma teoria bastante respeitada entre pesquisadores da área, a Teoria Ecológica de Bronfenbrenner (1986), defende, há muito, a ideia de que o desenvolvimento humano deve ser estudado dentro de seu contexto. Essa perspectiva ecológica também tem se refletido no campo da avaliação neuropsicológica, que inclui diferentes fontes de informação a respeito do comportamento da criança (e.g., Seabra-Santos \& Gaspar, 2012), usualmente, pais/responsáveis e professores, por serem as pessoas que mais convivem com as crianças. Entretanto, os resultados das pesquisas com pais e professores têm mostrado diferenças entre as avaliações desses grupos. Uma pesquisa realizada por León, Rodriguez, Seabra e Dias (2013) a respeito da relação entre $\mathrm{FE}$ e desempenho escolar em crianças de 6 a 9 anos revelou diferenças na perspectiva de avaliação de pais e professores, conforme respostas a uma escala de avaliação de FE, em todas as subescalas, com exceção da subescala de Memória de Trabalho. Adicionalmente, segundo Seabra-Santos e Gaspar (2012), pesquisas realizadas sobre a concordância de informantes que são focadas em problemas de comportamento apresentam correlações baixas entre os informantes, havendo mais concordância nas percepções de comportamentos externalizantes do que internalizantes.

Além dessas dificuldades, a essência dinâmica do funcionamento das FE torna a sua avaliação clínica um desafio (Denckla, 1994). Em uma revisão sistemática com 106 estudos sobre conceitualização e operacionalização das funções executivas, Baggetta e Alexander (2016) encontraram 109 diferentes tarefas individuais e 11 baterias de testes para avaliar funções executivas. Conforme descrito por Dawson e Guare (2010), esse desafio fica ainda maior quando se avalia crianças, primeiramente, porque os testes padronizados para avaliação de FE foram originalmente desenvolvidos para adultos, sem apelos para crianças ou informações normativas suficientes. Em segundo lugar, esses testes foram desenvolvidos para uma população clínica, não sendo sensíveis para detectar o desenvolvimento progressivo das habilidades executivas em crianças. Como uma terceira dificuldade, os autores supracitados referem que as tarefas nos testes frequentemente requerem que se usem outras habilidades no exercício das FE, como linguagem, memória ou coordenação motora, fato que pode confundir os resultados se a criança tiver algum déficit nessas habilidades. Finalmente, alguns autores (e.g., Isquith, Crawford, Espy, \& Gioia, 
2005) têm questionado se haveria validade ecológica nas mensurações clínicas das FE. Em decorrência dessa gama de dificuldades, a avaliação das FE necessita ir além da aplicação formal da bateria de testes neuropsicológicos. Para enriquecer a coleta de informações em diferentes contextos, dois inventários destinados a pais (ou responsáveis) e professores têm sido utilizados em pesquisas e na prática clínica no Brasil: o Behavior Rating Inventory of Executive Function - BRIEF (Gioia, Isquith, Guy, \& Kenworthy, 2000) e o Inventário de Funcionamento Executivo Infantil - IFERA-I (Trevisan, Berberian, Dias, \& Seabra, 2012).

O presente estudo tem como objetivo geral investigar a associação entre as avaliações das FE de alunos do $5^{\circ}$ ano por pais e professores, avaliadas por meio dos instrumentos BRIEF e IFERA-I, e o desempenho escolar na disciplina de matemática. A faixa etária do $5^{\circ}$ ano foi escolhida porque corresponde a um período crítico, de transição entre os níveis I e II do ensino fundamental, em que a escola exige maior autonomia e independência dos alunos e, consequentemente, existe uma maior demanda das FE. Nesse período, observa-se um aumento das dificuldades na aprendizagem, indisciplina, desinteresse por parte dos alunos e queda do rendimento escolar (Borges, 2015). Além disso, a revisão da literatura mostrou grande número de pesquisas relacionando $\mathrm{FE}$ e desempenho acadêmico em crianças da educação infantil ao $3^{\circ}$ ano, mas poucos estudos fazendo essa investigação com crianças mais velhas.

De forma mais específica, este trabalho buscou investigar as seguintes perguntas de pesquisa: há correlação entre as avaliações de pais e professores nos instrumentos IFERA-I e BRIEF com o desempenho na disciplina de matemática? Há diferenças significativas nas avaliações de pais e professores em ambos os instrumentos?

Baseada na literatura da área, a hipótese deste estudo é que serão encontradas correlações significativas entre as FE e o desempenho na matemática, e que as avaliações do grupo de professores deverão apresentar diferenças importantes em relação ao grupo de pais. Os resultados deste estudo buscam contribuir para o refinamento da compreensão sobre as relações entre o funcionamento das FE e o desempenho em matemática, bem como para a investigação sobre as diferenças nas avaliações dos informantes, a saber, pais e professores. Tais conhecimentos são de fundamental importância para trazer subsídios para a criação de novos instrumentos de avaliação e intervenção em FE, bem como de instrumentos de intervenções psicopedagógicas que busquem auxiliar alunos a superarem possíveis dificuldades na matemática.

\section{Método}

\section{Participantes}

Participaram deste estudo pais/responsáveis e professores de 47 crianças, com idade entre 10 e 11 anos, matriculadas no $5^{\circ}$ ano em uma escola da rede particular de ensino, no interior do estado de São Paulo, caracterizada por atender crianças de nível socioeconômico médio a médio-alto. A escola foi escolhida por conveniência e, de acordo com os prontuários escolares e relato da coordenação da escola, nenhuma das crianças da amostra apresentava diagnóstico de transtorno do neurodesenvolvimento e/ ou deficiências visuais/auditivas não corrigidas.

\section{Instrumentos}

Foram utilizados dois instrumentos de avaliação de funções executivas, BRIEF e IFERA-I, que refletem aspectos da vida diária das crianças, visando quantificar e qualificar dificuldades executivas, com enfoque na funcionalidade. O BRIEF é uma escala ecológica para avaliação das FE em crianças e adolescentes, desenvolvido por Gioia et al. (2000), traduzido e adaptado para o português por Carim et al. (2012). Na versão utilizada para o presente estudo, esse instrumento apresenta-se como questionário com 86 questões, que avalia o comportamento das FE em casa e no ambiente escolar, devendo ser respondido por pais (ou responsáveis) e professores de crianças e adolescentes. Cada respondente deve graduar qual a frequência com que a criança apresenta determinado comportamento usando uma escala Likert com opções "nunca é um problema", "às vezes é um problema" e "constantemente é um problema". Na tabulação, a cada uma das opções é atribuído um valor de 1 a 3 , respectivamente. Portanto, quanto maior o escore gerado, maior o relato de dificuldades em FE. O resultado do BRIEF oferece uma mensuração global das FE e dois índices: regulação de comportamento (que inclui os domínios inibição, flexibilidade e controle emocional) e índice de metacognição (que abrange os domínios iniciativa, memória operacional, planejamento/organização, organização do material e monitoramento). Todas as medidas do BRIEF podem ser convertidas em escores $\mathrm{T}$ de acordo com a normatização original americana, o que possibilita a comparação do sujeito com o seu grupo normativo (Carim et al., 2012). Entretanto, no presente estudo, somente foram considerados os pontos brutos nas análises estatísticas, uma vez que até o momento não existe um manual de normatização brasileira desse inventário.

O IFERA-I é um instrumento desenvolvido por Trevisan e Seabra (2012), com base na Childhood Executive Function Inventory (Thorell \& Nyberg, 2008), que também deve ser respondido por pais e professores. É constituído por 28 itens divididos em cinco subescalas: Memória de Trabalho, Controle Inibitório, Flexibilidade, Aversão à Demora e Regulação. Cada item é avaliado dentro de uma escala Likert com opções "definitivamente não é verdadeiro", "não é verdadeiro", "é parcialmente verdadeiro", "é verdadeiro" e "definitivamente é verdadeiro", que recebem pontuação de 1 a 5 , respectivamente (León et al., 2013). Escores mais altos também são indicativos de maiores dificuldades. Como no caso do BRIEF, pelo 
fato de ainda não haver normatização brasileira para o IFERA-I, foram utilizados os pontos brutos nas análises estatísticas por meio das médias das pontuações em cada subescala.

\section{Procedimentos}

Inicialmente, distribuiu-se a 80 pais e responsáveis de três turmas do $5^{\circ}$ ano os Termos de Consentimento Livre e Esclarecido, com intuito de informá-los a respeito dos objetivos do estudo e de seu procedimento. Além disso, também lhes foi entregue os inventários BRIEF e o IFERA-I para que os preenchessem em casa. As três professoras das turmas foram instruídas para que respondessem aos questionários na escola. Além de assinarem o Termo de Consentimento Livre e Esclarecido e responderem aos questionários, os pais e responsáveis também autorizaram a coleta das notas bimestrais na disciplina de matemática de seus filhos junto banco de notas da escola.

Do BRIEF, foram respondidos 47 questionários pelos pais/responsáveis e pelas professoras. Dois participantes considerados outliers, com resultados discrepantes de mais de três desvios padrão acima da média, foram excluídos das análises. Em relação ao IFERA-I, pais e professores retornaram 42 e 46 questionários, respectivamente. Os dados do desempenho em matemática foram coletados nos boletins escolares fornecidos pelo banco de notas, sendo representados pela média das notas de matemática nos três bimestres cursados até o momento da aplicação dos instrumentos.

\section{Análise de dados}

A tabulação do BRIEF foi realizada seguindo critérios descritos na dissertação de mestrado de Carim (2010). Para a tabulação do IFERA-I, utilizaram-se critérios especificados por Trevisan e Seabra (2012). As análises foram conduzidas por meio do SPSS Statistics 20 (Statistical Package for the Social Sciences). Como as distribuições mostram-se normais, foram conduzidas estatísticas paramétricas. Inicialmente, foi conduzida uma análise de correlação de Pearson entre as notas em matemática e as avaliações no BRIEF e IFERA-I respondidos por pais e professores. Para verificar possíveis diferenças significativas entre as avaliações dos informantes, utilizou-se o teste $\mathrm{t}$ de Student para amostras em pares, comparando as avaliações globais de pais e professores em ambos os instrumentos.

\section{Resultados}

Os resultados das variáveis notas e índices do BRIEF e IFERA-I constam na Tabela 1.

Tabela 1

Medidas Descritivas das Variáveis Notas e Índices do BRIEF e IFERA-I

\begin{tabular}{|c|c|c|c|c|c|}
\hline Variável & Média & $\begin{array}{l}\text { Valor Mínimo } \\
\text { Valor Máximo }\end{array}$ & $\begin{array}{l}\text { Desvio } \\
\text { Padrão }\end{array}$ & $\begin{array}{l}\text { Coeficiente de } \\
\text { Variação }\end{array}$ & $\mathrm{N}$ \\
\hline Notas & 7,1 & $3.8-9.4$ & 1,39 & $19,60 \%$ & 46 \\
\hline Índice Global BRIEF Professores & 98,38 & $73-157$ & 19,62 & $19,90 \%$ & 45 \\
\hline Índice Global BRIEF Pais & 117,38 & $78-194$ & 23,87 & $20,30 \%$ & 45 \\
\hline Índice Total IFERA-I Professores & 2,32 & $1.3-4.7$ & 0,82 & $35,30 \%$ & 46 \\
\hline Índice Total IFERA-I Pais & 2,46 & $1.1-4.7$ & 0,71 & $28,90 \%$ & 42 \\
\hline
\end{tabular}

Para investigar as associações entre as diversas subescalas do BRIEF e IFERA-I e o desempenho em matemática, foram conduzidas análises de Correlação de Pearson. Conforme mostra a Tabela 2, todas as subescalas do BRIEF apresentaram correlação negativa com o desempenho em matemática, tanto nas avaliações de pais como professores. Isso revela uma tendência de que, quanto maior a pontuação em ambas as escalas, ou seja, mais dificuldades associadas às $\mathrm{FE}$, tanto menor tende a ser o desempenho em matemática. As avaliações dos professores mostraram as maiores correlações, especialmente nas subescalas Memória Operacional $(r=-0,730)$, Monitoramento $(r=-0,718)$ e nos Índices de Metacognição $(r=-0,725)$ e Índice Global $(r=-0,724)$. Além disso, com exceção da subescala Organização de Materiais, todas correlações dos professores mostraram-se maiores do que as dos pais.

A Tabela 3 apresenta os resultados das correlações entre as respostas de pais e professores no IFERA-I e as notas dos alunos em matemática. Também, nesse instrumento, as avaliações de pais e professores mostram correlações negativas com o desempenho em matemática, mas as correlações entre professores mostram-se mais altas em todas as subescalas, especialmente em Memória de Trabalho $(r=-0,646)$, Flexibilidade $(r=-0,565)$, Controle Inibitório $(r=-0,499)$ e Índice Total $(r=-0,565)$. 
Tabela 2

Correlação entre Notas em Matemática e Subescalas do BRIEF Preenchidas por Pais ( $N=45)$ e Professores (N=45)

\begin{tabular}{|c|c|c|c|c|c|}
\hline $\begin{array}{l}\text { Subescalas do BRIEF } \\
\text { avaliadas por pais }\end{array}$ & & Notas & $\begin{array}{l}\text { Subescalas do BRIEF avaliadas } \\
\text { por professores }\end{array}$ & & Notas \\
\hline \multirow{2}{*}{ Inibição } & $r$ & $-0,329^{* *}$ & \multirow{2}{*}{ Inibição } & $r$ & $-0,647^{* *}$ \\
\hline & $p$ & 0,027 & & $p$ & 0,000 \\
\hline \multirow{2}{*}{ Controle Emocional } & $r$ & $-0,41$ & \multirow{2}{*}{ Controle Emocional } & $r$ & $-0,546^{* *}$ \\
\hline & $p$ & 0,789 & & $p$ & 0,000 \\
\hline \multirow{2}{*}{ Flexibilidade } & $r$ & $-0,169$ & \multirow{2}{*}{ Flexibilidade } & $r$ & $-0,656^{* *}$ \\
\hline & $p$ & 0,267 & & $p$ & 0,000 \\
\hline \multirow{2}{*}{ Iniciativa } & $r$ & $-0,512^{* *}$ & \multirow{2}{*}{ Iniciativa } & $r$ & $-0,575^{* *}$ \\
\hline & $p$ & 0,000 & & $p$ & 0,000 \\
\hline \multirow{2}{*}{ Memória Operacional } & $r$ & $-0,477^{* *}$ & \multirow{2}{*}{ Memória Operacional } & $r$ & $-0,730^{* *}$ \\
\hline & $p$ & 0,001 & & $p$ & 0,000 \\
\hline \multirow{2}{*}{ Monitoramento } & $r$ & $-0,465^{* *}$ & \multirow{2}{*}{ Monitoramento } & $r$ & $-0,718^{* *}$ \\
\hline & $p$ & 0,001 & & $p$ & 0,000 \\
\hline \multirow{2}{*}{ Organização de Materiais } & $r$ & $-0,586^{* *}$ & \multirow{2}{*}{ Organização de Materiais } & $r$ & $-0,558^{* *}$ \\
\hline & $p$ & 0,000 & & $p$ & 0,000 \\
\hline \multirow{2}{*}{ Planejamento/Organização } & $r$ & $-0,548^{* *}$ & \multirow{2}{*}{ Planejamento/Organização } & $r$ & $-0,690^{* *}$ \\
\hline & $p$ & 0,000 & & $p$ & 0,000 \\
\hline \multirow{2}{*}{ Índice de Metacognição } & $r$ & $-0,581^{* *}$ & \multirow{2}{*}{ Índice de Metacognição } & $r$ & $-0,725^{* *}$ \\
\hline & $p$ & 0,000 & & $p$ & 0,000 \\
\hline \multirow{2}{*}{$\begin{array}{l}\text { Índice de Regulação } \\
\text { de Comportamento }\end{array}$} & $r$ & $-0,200$ & \multirow{2}{*}{$\begin{array}{l}\text { Índice de Regulação } \\
\text { de Comportamento }\end{array}$} & $r$ & $-0,692^{* *}$ \\
\hline & $p$ & 0,188 & & $p$ & 0,000 \\
\hline \multirow{2}{*}{ Índice Global } & $r$ & $-0,477^{* *}$ & \multirow{2}{*}{ Índice Global } & $r$ & $-0,724^{* *}$ \\
\hline & $p$ & 0,000 & & $p$ & 0,000 \\
\hline
\end{tabular}

Nota. $r=$ coeficiente de correlação de Pearson; $p=$ nível de significância; ${ }^{* *}$ Correlação significante ao nível 0,01 (duas extremidades); ${ }^{*}$ Correlação significante ao nível 0,05 (duas extremidades)

Tabela 3

Correlação entre Notas em Matemática e Subescalas do IFERI Preenchidas por Pais (N=42) e Professores $(N=46)$

\begin{tabular}{|c|c|c|c|c|c|}
\hline $\begin{array}{c}\text { Subescalas do } \\
\text { IFERI avaliadas por pais }\end{array}$ & & Notas & $\begin{array}{c}\text { Subescalas do IFERI avaliadas } \\
\text { por professores }\end{array}$ & & Notas \\
\hline \multirow{2}{*}{ Controle Inibitório } & $r$ & $-0,332^{* *}$ & \multirow{2}{*}{ Controle Inibitório } & $r$ & $-0,499^{* *}$ \\
\hline & $p$ & 0,032 & & $p$ & 0,000 \\
\hline \multirow{2}{*}{ Flexibilidade } & $r$ & $-0,347^{* *}$ & \multirow{2}{*}{ Flexibilidade } & $r$ & $-0,565^{* *}$ \\
\hline & $p$ & 0,024 & & $p$ & 0,000 \\
\hline \multirow{2}{*}{ Memória de Trabalho } & $r$ & $-0,578^{* *}$ & \multirow{2}{*}{ Memória de Trabalho } & $r$ & $-0,646^{* *}$ \\
\hline & $p$ & 0,000 & & $p$ & 0,000 \\
\hline \multirow{2}{*}{ Regulação } & $r$ & $-0,294$ & \multirow{2}{*}{ Regulação } & $r$ & $-0,497^{* *}$ \\
\hline & $p$ & 0,058 & & $p$ & 0,000 \\
\hline \multirow{2}{*}{ Aversão à Demora } & $r$ & $-0,136$ & \multirow{2}{*}{ Aversão à Demora } & $r$ & $-0,361^{* *}$ \\
\hline & $p$ & 0,391 & & $p$ & 0,014 \\
\hline \multirow{2}{*}{ Total } & $r$ & $-0,391^{* *}$ & \multirow{2}{*}{ Total } & $r$ & $-0,565^{* *}$ \\
\hline & $p$ & 0,011 & & $p$ & 0,000 \\
\hline
\end{tabular}

Nota. $r=$ coeficiente de correlação de Pearson; $p=$ nível de significância; ${ }^{* *}$ Correlação significante ao nível 0,01 (duas extremidades); ${ }^{*}$ Correlação significante ao nível 0,05 (duas extremidades) 
Comparando as correlações que os dois instrumentos apresentaram com notas em matemática, observa-se que o BRIEF apresentou maiores correlações, ainda que a maior parte das correlações do IFERA-I também tenham sido significativas. Destaca-se o fato de que, tanto entre pais como entre professores, as maiores correlações ocorreram na subescala de Memória Operacional ou de Trabalho. As menores correlações ocorreram, para o IFERA-I, na subescala Aversão à Demora $(r=-0,136)$, e, para o BRIEF, em Controle Emocional $(r=-0,41)$, Flexibilidade $(r=-0,169)$, e no Índice de Regulação de Comportamento $(r=-0,200)$, avaliados pelos pais. A análise do teste $t$ para amostras em pares, conduzida para verificar as diferenças entre as avaliações de pais e professores em cada instrumento, mostrou diferenças significativas no instrumento BRIEF, $\operatorname{com} t(44)=6,69, p<0,001 \mathrm{e}$ efeito de magnitude grande, $d=0,71$, como também no IFERA-I, com $t(41)=-2,48, p=0,017$, com magnitude de efeito média, $d=0,36$. Em ambos os instrumentos os professores relataram menos dificuldades do que os pais.

\section{Discussão}

As FE, responsáveis pelo controle de pensamentos e ações, têm se mostrado relacionadas a desfechos acadêmicos, tais como o desempenho escolar em matemática. Porém, as avaliações de FE via relatos de pais e professores tendem a apresentar baixa concordância entre si. De modo a compreender melhor tais aspectos, este estudo buscou analisar a relação de FE com desempenho em matemática, bem como verificar a relação entre os relatos de diversos respondentes.

Confirmando a hipótese inicial, houve correlações negativas das notas em matemática com o BRIEF e o IFERA-I, sugerindo que crianças com piores desempenhos em matemática tenderam a ser avaliadas por pais e professores como tendo mais dificuldades em FE. Além disso, entre as FE investigadas pelos instrumentos, os resultados mostraram as maiores correlações entre memória de trabalho e desempenho matemático, em ambos instrumentos. Tal fato mostra-se congruente com estudos prévios (Bull, \& Lee, 2014; Cragg, \& Gilmore, 2014; Peng, Namkung, Barnes, \& Sun, 2016; Wu, et al., 2017; Viterbori, Usai, Traverso, \& De Franchis, 2015), bem como com uma metanálise com 111 estudos, que revelou a memória de trabalho como um forte preditor do desempenho em matemática (Friso-Van Den Bos, Van Der Ven, Kroesbergen, \& Van Luit, 2013), sendo o executivo central o componente mais importante. De acordo com Cragg, Keeble, Richardson, Roome e Gilmore (2017), a memória de trabalho é necessária para ativar e evocar fatos matemáticos armazenados na memória de longo prazo, contribuindo com variância exclusiva tanto para o desempenho matemático global, quanto para conhecimento de fatos matemáticos, habilidades procedimentais e compreensão de conceitos.
Adicionalmente, controle inibitório e flexibilidade foram outros componentes das FE que apresentaram altas correlações com o desempenho em matemática, porém, mais baixas que a memória de trabalho. Essa constatação também é congruente com a metanálise supracitada de Friso-Van Den Bos et al. (2013), que mostrou que inibição e flexibilidade são funções menos importantes para o desempenho matemático do que a memória de trabalho. Outros estudos sugerem que as FE de inibição e flexibilidade podem trazer variância no desempenho da matemática quando são investigados em análises de regressão de forma independente, mas não quando a memória de trabalho é incluída no modelo (Bull \& Lee, 2014).

Tais dados são relevantes, pois, para que o ensino da matemática ocorra de forma eficaz, é de suma importância que se conheça a gama de fatores que contribuem para o desempenho nessa disciplina. Estudos apontam o conhecimento de fatos matemáticos, as habilidades de procedimento, o entendimento conceitual e as habilidades executivas como preditores do desempenho em matemática (Cragg et al., 2017). Logo, se FE e, especialmente, memória de trabalho têm se revelado preditores importantes de matemática, é fundamental que práticas de intervenção regulares ou remediativas possam incluir componentes executivos, de modo a possibilitar a aprendizagem dessa disciplina.

Em concordância com estudos na literatura, diferenças significativas entre as respostas de pais e professores aos inventários foram verificadas em ambos os instrumentos, sendo no BRIEF, com efeito de magnitude grande e, no IFERA-I, médio. As respostas dos professores obtiveram correlações mais altas com o desempenho matemático em todas as escalas, exceto na organização de materiais do BRIEF. Por sua vez, os pais parecem ter sido mais rigorosos na avaliação do comportamento de seus filhos. Para entender esses achados, alguns autores sugerem que professores possuem melhores condições para avaliar comportamentos relacionados a habilidades cognitivas, já que possuem uma base normativa de comparação das habilidades e comportamentos esperados para determinada faixa etária e gênero. Por outro lado, pais tendem a estar mais familiarizados com alguns comportamentos específicos que se manifestam de forma importante no ambiente de casa, como, por exemplo, a organização de materiais. Consequentemente, esses comportamentos podem ser mais bem avaliados pelos pais (León et al., 2013; Seabra-Santos \& Gaspar, 2012).

Deve-se considerar, ainda, que os próprios professores eram os responsáveis pelas notas escolares dos alunos, por meio de um processo objetivo de avaliação, as provas escolares, o que também pode ter contribuído para a maior correlação das notas com os relatos dos professores. A literatura mostra que pais tendem a avaliar problemas de comportamento de forma mais severa que professores (Major, 2011; Marzocchi et al., 2004; Winsler \& Wallace, 2002). Essa tendência também foi 
observada na presente pesquisa. Alguns autores ressaltam que a marcante diferença entre os ambientes escolares e domésticos pode ser responsável por diferenças no comportamento das crianças nos dois ambientes e, consequentemente, pelas diferenças nas avaliações de pais e professores (Seabra-Santos \& Gaspar, 2012). Tal fato reforça a importância de englobar múltiplos informantes, além da avaliação do próprio desempenho, especialmente na avaliação de crianças (Martoni, Trevisan, Dias, \& Seabra, 2016; Seabra-Santos \& Gaspar, 2012).

\section{Considerações Finais}

Esse foi o primeiro estudo brasileiro realizado visando comparar as associações entre dois inventários de FE, BRIEF e IFERA-I, e o desempenho escolar em matemática. Em concordância com a literatura, o presente estudo mostrou que existe uma associação de correlação significativa entre as notas na disciplina de matemática e a pontuação de pais e professores nos Inventários do BRIEF e IFERA-I. Ou seja, as piores notas de matemática estão associadas às maiores pontuações, que, nesses instrumentos, indicam maiores dificuldades executivas. Além disso, este estudo também mostrou que os inventários respondidos por professores apresentaram maiores correlações com as notas de matemática do que os respondidos por pais. Tal fato, como discutido anteriormente, pode refletir condições diferenciadas de avaliação das crianças em ambientes distintos, reiterando a importância de obter diferentes fontes de informação para a avaliação, especialmente, de crianças pequenas.

Ressalta-se, porém, que o estudo apresentou limitações, como, por exemplo, o número pequeno de alunos investigados, além do fato de que tais alunos pertenciam a um grupo restrito de uma mesma série e da mesma escola, o que não permite a generalização dos resultados para a população geral. Destaca-se, ainda, o fato de que pais/responsáveis preencheram os questionários em casa, o que diminui o controle sobre quem de fato respondeu ao questionário ou sobre possíveis dificuldades de compreensão das questões. É importante ressaltar que a avaliação de funções executivas foi feita exclusivamente por meio de relatos de terceiros, no caso, pais e professores, o que pode gerar vieses tipicamente relacionados a essa forma de coleta de informações. Sugere-se que futuros estudos acrescentem avaliações de funções executivas de outra natureza, como testes de desempenho. Adicionalmente, é recomendável aumentar o número de participantes, incluindo outros tipos de escola e níveis socioeconômicos, para que se torne possível a condução de análises de regressão e, consequentemente, a investigação de quais subescalas e índices do BRIEF e IFERA-I mais contribuem para a variância das notas de matemática.

\section{Agradecimentos}

Não há menções.

\section{Financiamento}

A presente pesquisa não recebeu nenhuma fonte de financiamento sendo custeada com recursos dos próprios autores.

\section{Contribuições dos autores}

Todos os autores contribuíram substancialmente para a elaboração do delineamento da pesquisa, análise e interpretação dos dados, bem como, para a revisão textual e aprovação da versão final deste estudo. Todos os autores assumem responsabilidade pública pelo conteúdo do manuscrito.

\section{Disponibilidade dos dados e materiais}

Todos os dados e sintaxes gerados e analisados durante esta pesquisa serão tratados com total sigilo devido às exigências do Comitê de Ética em Pesquisa em Seres Humanos. Porém, o conjunto de dados e sintaxes que apoiam as conclusões deste artigo estão disponíveis mediante razoável solicitação ao autor principal do estudo.

\section{Conflito de interesses}

Os autores declaram que não há conflitos de interesses.

\section{Referências}

Baggetta, P., \& Alexandrer, P.A. (2016). Conceptualization and operationalization of executive function. Mind, Brain, and Education, 10, 1033. doi: $10.1111 / \mathrm{mbe} .12100$

Blair, C., \& Razza, R. (2007). Relating effortful control executive function, and false belief understanding to emerging math and literacy ability in kindergarten. Child Development, 78, 647-663. doi: 10.1111/j.1467-8624.2007.01019.x

Blair, C., Protzko, J., \& Ursache, A. (2011). Self-regulation and early literacy. Em S. Neuman, \& D. Dickinson, Handbook of early literacy research (vol 3) (p. 20-35). New York, NY: Guilford.

Borges, R. (2015). Desafios do educador na transição do quinto para o sexto ano nas escolas públicas do estado de SP: Uma proposta de formação (Dissertação de mestrado). São Paulo, SP. Recuperado de https://tede2.pucsp.br/handle/handle/10254

Bronfenbrenner, U. (1986). The ecology of the family as a context for human development. Developmental Psychology, 22, 723-742. doi: $10.1037 / 0012-1649.22 .6 .723$ 
Bull, R., \& Lee, K. (2014). Executive functioning and mathematics achievement. Child Development Perspectives, 8(1), 36-41. doi: 10.1111/ cdep. 12059

Carim, D. B. (2010). Tradução e adaptação para o português do instrumento "Behavior Rating Inventory of Executive Function" (Dissertação de mestrado). UNIFESP, São Paulo, SP.

Carim, D. B., Miranda, M. C., \& Bueno, O. (2012). Tradução e adaptação para o português do Behavior Rating Inventory of Executive Function - BRIEF. Psicologia: Reflexão e Crítica, 25(4), 653-661. doi: 10.1590/S0102-79722012000400004

Clements, D., Sarama, J., \& Germeroth, C. (2016). Learning executive function and early mathematics: Directions of causal relations. Early Childhood Research Quarterly, 36, 79-90. doi: 10.1016/j.ecresq.2015.12.009

Cragg, L., \& Gilmore, C. (2014). Skills underlying mathematics: The role of executive function in the development of mathematics proficiency. Trends in Neuroscience and Education, 3, 63-68. doi: 10.1016/j.tine.2013.12.001

Cragg, L., Keeble, S., Richardson, S., Roome, H., \& Gilmore, C. (2017). Direct and indirect influences of executive functions on mathematics achievement. Cognition, 162, 12-26. doi: 10.1016/j.cognition.2017.01.014

Dawson, P., \& Guare, R. (2010). Executive Skills in Children and Adolescents. New York, NY: The Guilford Press.

Denckla, M. B. (1994). Measurement of executive function. Em G. R. Lyon (Org.), Frames of reference for the assessment of learning disabilities: New views on measurement issues (117-142). Baltimore: Paul H. Brookes.

Diamond, A. (2013). Executive Functions. Annual Review of Psychology, 64, 135-168. doi: 10.1146/annurev-psych-113011-143750

Friso-Van Den Bos, I., Van Der Ven, S. H., Kroesbergen, E. H., \& Van Luit, J. E. (2013). Working memory and mathematics in primary school children: A meta-analysis. Educational research review, 10, 29-44.

Gioia, G., Isquith, P., Guy, S., \& Kenworthy, L. (2000). Behavior rating inventory of executive function, professional manual. Lutz, FL: Psychological Assessment Resource.

Isquith, P., Crawford, J., Espy, K., \& Gioia, G. (2005). Assessment of executive function in pre-school aged children. Mental Retardation and Developmental Disabilities Research Reviews, 11, 209-215. doi: 10.1002/mrdd.20075

Lan, X., Legare, C., Ponitz, C., Li, S., \& Morrison, F. (2011). Investigating the links between the subcomponents of execuitve functions and academic achievement: A cross-cultural analysis of chinese and American pre-schoolers. Journal of Experimental Child Psychology, 108, 677- 692. doi: 10.1016/j.jecp.2010.11.001

León, C., Rodriguez, C., Seabra, A. G., \& Dias, N. (2013). Funções executivas e desempenho escolar em crianças de 6 a 9 anos de idade. Revista Psicopedagogia, 30(92), 113-120. Recuperado de http://pepsic.bvsalud.org/scielo.php?script=sci_arttext\&pid $=$ S0103-84862013000200005

Major, S. O. (2011). Avaliação de aptidões sociais e problemas de comportamento em idade pré-escolar. Estudo exploratório com crianças portuguesas (Dissertação de mestrado). Faculdade de Psicologia e de Ciências da Educação, Universidade de Coimbra, Portugal.

Martoni, A. T., Trevisan, B. T., Dias, N. M. \& Seabra, A. G. (2016). Funções executivas: Relação entre relatos de pais, professores e desempenho de crianças. Temas em Psicologia,(24)1, 173-188. doi: 10.9788/TP2016.1-12

Marzocchi, G. M., Capron, C., Pietro, M., Tauleria, E. D., Duyme, M., ... Frigerio, A. (2004). The use of the Strengths and Difficulties Questionnaire (SDQ) in Southern European countries. European Child E Adolescent Psychiatry, 13(Suppl. 2), 40-46. doi: 10.1007/ s00787-004-2007-1

Miyake, A., Friedman, N., Emerson, M., Wotzki, A., Howerter, A., \& Wager, T. (2000). The unity and diversity of executive functions and their contributions to complex "frontal lobe" tasks: A latent variable analysis. Cognitive Psychology, 41, 49-100. doi: 10.1006/ cogp.1999.0734

Miyake, A., \& Friedman, N. P. (2012). The Nature and Organization of Individual Differences in Executive Functions: Four General Conclusions. Current Directions in Psychological Science, 21(1), 8-14. doi: 10.1177/0963721411429458.

Monnete, S., Bigras, M., \& Guay, M. C. (2011). The role of executive functions in school achievement at the end og grade 1. Journal of Experimental Child Psychology, 109(02), 158-173. doi: 10.1016/j.jecp.2011.01.008

Passolunghi, M., \& Lanfranchi, S. (2012). Domain-specific and domain-general precursors of mathematical achievement: A longitudinal study form kindergarten to first grade. British Journal of Educational Psychology, 82, 42-63. doi: 10.1111/j.2044-8279.2011.02039.x

Peng, P., Namkung, J., Barnes, M., \& Sun, C. (2016). A meta-analysis of mathematics and working memory: Moderating effects of working memory domain, type of mathematics skill, and sample characteristics. Journal of Educational Psychology, 108(4), 455.

Seabra-Santos, M., \& Gaspar, M. (2012). Pais, educadores e testes: Estão de acordo na avaliação de crianças pré-escolares? Psicologia: Reflexão e Crítica, 2, 203-211. Recuperado de http://www.scielo.br/pdf/prc/v25n2/a01v25n2.pdf

Thorell, L., \& Nyberg, L. (2008). The childhood executive functioning inventory (CHEXI): A new rating instrument for parents and teachers. Developmental Neuropsychology, 33, 536-52. doi: 10.1080/87565640802101516

Trevisan, B., Berberian, A., Dias, N., \& Seabra, A. G. (submetido). Development and psychometric properties of the Difficulties in Executive Functions, Regulation and Delay Aversion Inventory (IFERA-I).

Trevisan, B. T., \& Seabra, A. G. (2012). Inventário de Dificuldades em Funções Executivas, Regulação e Aversão ao Adiamento: Versão para crianças e Adolescentes (IFERA-I). (Teste não publicado). São Paulo: Universidade Presbiteriana Mackenzie.

Van Der Ven, S. (2011). The structure of executive functions and relations to early mathematics learning. Utrecht, Germany: Labor Grafimedia BV.

Viterbori, P., Usai, M., Traverso, L., \& De Franchis, V. (2015). How preschool executive functioning predicts several aspects of math achievement in Grades 1 and 3: A longitudinal study.Journal of Experimental Child Psychology, 140, 38-55. doi: 10.1016/j.jecp.2015.06.014

Winsler, A., \& Wallace, G. L. (2002). Behavior problems and social skills in preschool children: Parent-teacher agreement and relations with classroom observations. Early Education \& Development, 13(1), 41-58. doi: 10.1207/s15566935eed1301_3

Wu, S. S., Chen, L., Battista, C., Watts, A. K. S., Willcutt, E. G., \& Menon, V. (2017). Distinct influences of affective and cognitive factors on children's non-verbal and verbal mathematical abilities. Cognition, 166, 118-129.

Zelazo, P. D., \& Muller, U. (2002) Executive Function in Typical and Atypical development. In Blackwell Handbook of Childhood Cognitive Development, Wiley Online Library, 445-469. doi: 10.1002/9780470996652.ch20 
Zelazo, P., \& Muller, U. (2011). Executive function in typical and atypical development. Em U. Goswami, The Wiley-Blackwell handbook of childhood cognitive development (2nd ed.). Hoboken, NJ: Wiley-Blackwell.

Zimmerman, B. (2002). Achieving academic excellence: A self-regulatory experience. Em M. Ferrar (Ed.), The pursuit of excellence through education (85-110). Mahwah, NJ: Eribaum.

\section{Sobre as autoras}

Cristiane Marx Flor é psicóloga (USP) e neuropsicóloga (FMUSP). Fez mestrado em Neurociência Aplicada à Educação e Estudos da Criança na Universidade de Leiden. Atualmente, é doutoranda no Programa de Pós-Graduação em Distúrbios do Desenvolvimento da Universidade Presbiteriana Mackenzie, onde é bolsista da CAPES.

Ana Paula Soares de Campos é pedagoga pelo UNISAL e psicopedagoga pela Universidade São Marcos. É mestre e doutoranda pelo Programa de Pós-Graduação em Distúrbios do Desenvolvimento da Universidade Presbiteriana Mackenzie (UPM). Atua como docente no ensino superior nos cursos de pedagogia, psicopedagogia e licenciaturas da Universidade Presbiteriana Mackenzie.

Suzete Araújo Água Maia é pedagoga e mestre em Educação pelo Centro Universitário Adventista de São Paulo (UNASP). É doutoranda pelo Programa de Pós-Graduação em Distúrbios do Desenvolvimento da Universidade Presbiteriana Mackenzie. Atua como diretora de graduação na Faculdade Adventista de Hortolândia (FAH/UNASP-HT).

Alessandra Gotuzo Seabra é psicóloga e doutora em Psicologia Experimental pela Universidade de São Paulo. Atua como docente e pesquisadora do Programa de Pós-graduação em Distúrbios do Desenvolvimento da Universidade Presbiteriana Mackenzie. Bolsista de Produtividade do CNPq.

\section{Como citar este artigo}

Flor et al. (2020). Funções Executivas: Correlação entre dois Inventários e Desempenho Acadêmico em Matemática. Avaliação Psicológica, 19(3), 268-276. http://dx.doi.org/10.15689/ap.2020.1903.15546.05 